\title{
PERFORMANCE EVALUATION OF AN ADAPTABLE inVANETS-BASED INTERSECTION TRAFFIC CONTROL
}

\author{
Hadeel A. Tabaza, Iman Saeed and Mourad Elhadef ${ }^{1}$ \\ College of Engineering, Abu Dhabi University, Abu Dhabi, UAE \\ ${ }^{l}$ Ph.D.
}

\begin{abstract}
With the recent advances in intelligent vehicles ad-hoc networks (inVANETs), the traditional traffic control problem at intersections is being revisited to cope with the future intelligent transportation systems. In this paper, we introduce the performance evaluation of a centralized inVANETs-based intersection traffic control protocol. Extensive simulations have been conducted using various real traffic scenarios to evaluate the efficiency and the dynamics of the newly proposed traffic control approach that gives the intelligent vehicles full autonomous control of crossing intersections using vehicle-to-vehicle and vehicle-to-infrastructure communications. Simulation results using the tools OMNet++, VEINS, and SUMO showed that the new approach is a viable alternative and addition to the existing traffic control approaches.
\end{abstract}

\section{KEYWORDS}

Smart cities, Intelligent Transportation Systems, Intersection Traffic Control, Distributed Algorithms, Intelligent Vehicles ad-hoc networks (inVANETs)

\section{INTRODUCTION}

An important part of Intelligent Transportation System (ITS) is the intersection traffic control, as it needs to adapt to the increasing number of congestions as well as emergencies and accidents (Wei 2014), (Srinivasan 2006), (Baras et al. 2017). Many applications for ITS were proposed such as collision avoidance, traffic surveillance which make the way to the next generation of smart cities. Numerous solutions were proposed to control intersections automatically. The conventional solutions mostly concentrated on improving the scheduling of the traffic lights to reduce congestion as well as increasing the vehicles flow on the intersections, (Chen 2010). The new approaches focus on more intelligent scheduling of traffic lights depending mainly on computational intelligence for example like evolutionary genetic algorithm (Chen 2010), (Zhao 2012), machine learning (Gokulan 2010), fuzzy logic (Qiao 2011), (Lertworawanich 2011), (Gokulan 2010), and neural networks (Wei 2014), (Qiao 2011). For more details the readers can read this survey (Bhatt 2010), (Hassan and Elhadef 2018), (Saeed and Elhadef 2017).

The insufficiencies were shown by Wu et al (Wu 2014) in this approach such as deciding the optimal time for the green light knowing the subtleties of the flow of the traffic. Moreover, the algorithms of computational intelligence consume time thus aren't suitable for real time traffic control. Advanced communication and sensing technologies were proposed in (Srinivasan 2006), (Lee 2010), (Zhou 2010) which are being used to allow real time traffic response for green light traffic control that uses a type of control strategy deduced from preselected logic rules and traffic data to schedule traffic lights. Another approach was the trajectory maneuver has been discussed in (Lee 2010), (Zhou 2010), (Tubaishat 2009), (Glaser et al. 2010). This approach is built on an intersection controller that handles the trajectories of the vehicles knowing the conditions of the nearby vehicles to prevent any possibility for an overlap. In this approach, the vehicles are able to move without the need to stop, and this leads to enhancing the system's efficiency. Numerous ways have been studied in order to calculate the ideal trajectories, such as fuzzy logic (Raravi 2007), merging (Dresner 2008), cell-based (Bulgannawar 1995), scenario-driven (Milans et al. 2010), global adjusting, and exception handling (Srinivasan 2006). There are some insufficiencies in the trajectory maneuver approach such as the trajectory calculation complexity and being reliant on a centralized controller causes this approach to be costly, and likely to single point of failure. 
In this paper, we are going to evaluate the proposed novel adaptable centralized intersection control for intelligent transportation systems, which relies on inVANETs. The intersection control algorithm that is based on inVANETs contains two tasks: the vehicleTask, and the controllerTask. The two tasks work with each other using the exchange of messages to let the vehicles cross an intersection with no conflicts at all. This work is based on the centralized algorithm that was developed in ( $\mathrm{Wu} 2014)$ that considers the existence of traffic controller that its role to give the privilege of passing the intersection to the vehicles requesting to cross. The centralized approach considers that if the controller gives the passing to a queue of vehicles on the intersection, then it waits until the release message is received from the last vehicle in the queue, or in real life situations there might be a delay for vehicles due to bottleneck congestion, or accidents, which make using this centralized approach impractical. The traditional scheduling of traffic light overcomes the situation by changing the lights to red for a certain amount of time to allow the vehicles to pass while resolving the situation. The adaptable inVANETs work proposes a solution to solve the weakness in the original centralized approach, which adapts this approach to real scenarios. In the controller-based approach, an algorithm for locks is being used to give or reject access to the intersection the waiting vehicles can cross the intersection when the controller has locked the lanes that are conflicting successfully. When a vehicle reaches the queuing area, it sends a request to the controller to lock the conflicting lanes. The controller, which manages the locks, will reply to the vehicle with a message to give it access to the intersection. The objective of the controller is to guarantee the efficiency of the mutual exclusion as well as maximize the number of vehicles passing in the intersection simultaneously. The paper is organized in this approach. In Section 2, we discuss preliminary definitions, and explain the system model. In Section 3, we discuss the algorithm, the messages, and other details of the new adaptable inVANETs-based intersection control. In Section 4, we discuss the application of algorithm in Abu Dhabi, and in Section 5 we discuss the performance evaluation results. In Section 6, we conclude the paper.

\section{PRELIMINARY}

The adopted system model was developed by (Wu 2014). Consider this example of an intersection having four directions (Figure 1), and the lanes are numbered from 10 to 17. The core area is the area of the small dashed square, and the larger area is the one called the queuing area. Each vehicle will have the state of CROSSING in the core are, and the state will be either WAITING or QUEUING in the queuing area. The rules of the traffic intersection state that the vehicles having crossing paths such as $l_{0}$, and $l_{6}$ should pass the intersection mutually exclusively. These lanes are supposed to be conflicting and represented by $\propto$. The vehicles having non-crossing paths such as $l_{0}$, and $l_{4}$ can pass the intersection at the same time. These lanes are considered to be concurrent and represented by $\approx$. Using this definition, we have $l_{0} \propto l_{6}$, and $l_{0} \approx l_{4}$. In $(\mathrm{Wu} 2014)$ the concurrency and conflict relationships were represented using a graph, which can be used with any type of intersection.

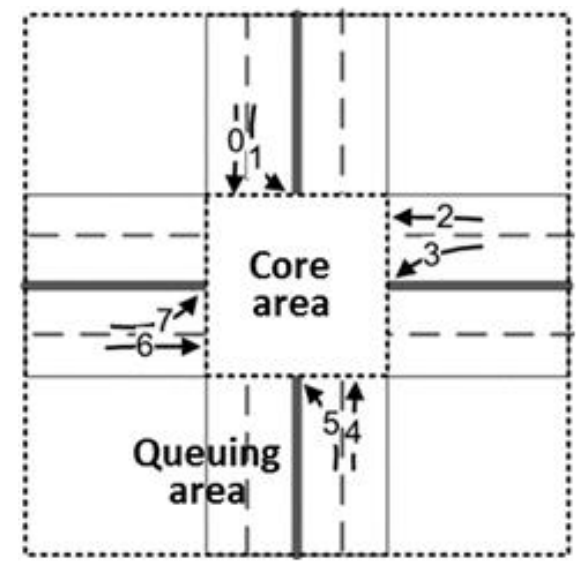

Figure 1. A Typical intersection

It is assumed that each vehicle has its own $I D$ and is self-driven. The vehicles count on their sensors, and other devices for navigation, and collision avoidance. In addition, each vehicle is able to detect the core, and queuing areas' limits using the positioning system of the vehicle such as GPS, as well as sensors placed at the limits of the areas. Vehicles have devices for wireless communication that allow the vehicle to communicate in 
vehicle-to-infrastructure communication or vehicle-to-vehicle communication. In addition, the vehicles are equipped with wireless FIFO channels. It is assumed that the message transmission is reliable, and the range of transmission is larger than the length of the queuing area. Therefore, all the vehicles in the queuing area creating a one-hop ad-hoc network, which allows any set of vehicles to directly communicate.

In Figure 2 you can see the transition of states of the vehicle. Any vehicle will start in the IDLE state if it's outside of the intersection area. The state of the vehicle will change when it enters the queuing area to WAITING. When the vehicle is in this state, it will request the access to the core area from the controller and waits for the approval from the controller. When the controller gives the access the vehicle, the state of the vehicles changes to QUEUING. The vehicles having the QUEUING state cross the core area in a first-in-first-out fashion. The vehicles that are delayed and cannot cross the core area in a specified certain time, their state will change to WAITING again. Moreover, the vehicle that change its position to another lane which is conflicting while being the QUEUING state, its state will also change to WAITING. Then the vehicle needs to send the request to cross again to the controller to cross the intersection. The vehicle state is changed to CROSSING when it enters the core area. Finally, when the vehicle crosses the core area, and leaves, the state is changed to IDLE.

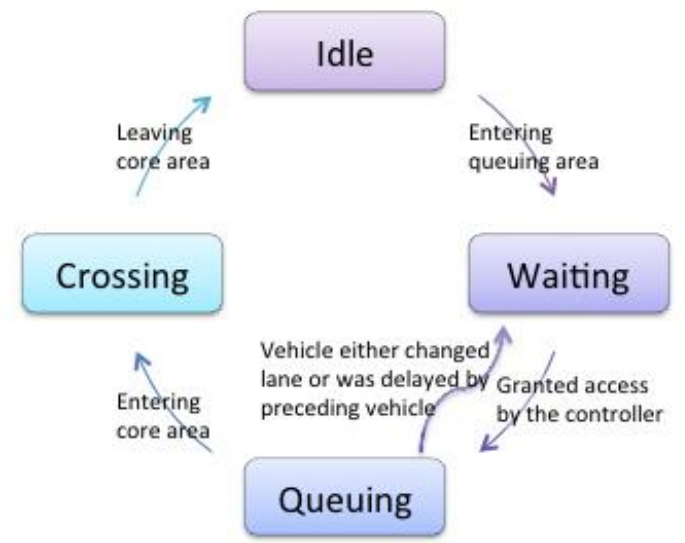

Figure 2. Vehicle State transition diagram

In (Wu 2014), they showed the intersection traffic control like a vehicle mutual exclusion for intersections (VMEI) problem, which says that at the intersection, each vehicle sends a request in whatever direction it desires. Therefore, the vehicles queue up at the equivalent lanes in the queuing area. The vehicles can pass the intersection in the same time if they are in the same lane or concurrent lanes. Also in (Wu 2014), the correctness properties are summarized in order to give any solution to the problem as in the terms of safety, liveness, and fairness, shown below.

Safety (mutual exclusion): at any given moment, in the core area, no conflicting vehicles can be in it. If there was more than one vehicle, these vehicles should be concurrent with each other.

Liveness (deadlock free): any vehicle in waiting state will be given the access to the core area in a finite time.

Fairness (starvation free): each vehicle should be able to pass, after a finite number of vehicles pass the intersection, and all the lanes share the same priority.

\section{AN ADAPTABLE INVANETS-BASED INTERSECTION TRAFFIC CONTROL ALGORITHM}

In (Wu 2014), the first algorithm that was developed to solve the VMEI problem was called the centralized algorithm. It considers the existence of a controller which its role to give privilege to requesting vehicles to pass the intersection. It is shown that the algorithm mentioned in (Wu 2014) does not satisfy the properties of safety, liveness, and fairness. There is an error in the locking mechanism that does not satisfy the safety property. This algorithm considers the existence of traffic controller that its role to give the privilege of passing the intersection to the vehicles requesting to cross. The centralized approach considers that if the controller gives the passing to a queue of vehicles on the intersection, then it waits until the release message is received from the last vehicle 
in the queue, or in real life situations there might be a delay for vehicles due to bottleneck congestion, or accidents, which make using this centralized approach impractical. The traditional scheduling of traffic light overcomes the situation by changing the lights to red for a certain amount of time to allow the vehicles to pass while resolving the situation.

The adaptable inVANET work proposes a solution to solve the weakness in the original centralized approach, which adapts this approach to real scenarios. In the controller-based approach, an algorithm for locks is being used to give or reject access to the intersection the waiting vehicles can cross the intersection when the controller has locked the lanes that are conflicting successfully. When a vehicle reaches the queuing area, it sends a request to the controller to lock the conflicting lanes. The controller, which manages the locks, will reply to the vehicle with a message to give it access to the intersection. The objective of the controller is to guarantee the efficiency of the mutual exclusion as well as maximize the number of vehicles passing in the intersection simultaneously.

The controller preserves one lock per lane, therefore, for the intersection shown in Figure 1 eight locks are going to be used. Since the safety property was not satisfied by (Wu 2014), the locking mechanism was improved and correcting, and still relies on the same idea of locking two conflicting lanes.

Table 1. The lane-locking scheme

\begin{tabular}{|l|l|l|l|l|l|l|l|l|}
\hline Vehicle's lane & $\mathbf{0}$ & $\mathbf{1}$ & $\mathbf{2}$ & $\mathbf{3}$ & $\mathbf{4}$ & $\mathbf{5}$ & $\mathbf{6}$ & $\mathbf{7}$ \\
\hline Lanes to be & 0 & 1 & 1 & 0 & 1 & 2 & 3 & 1 \\
locked & 5 & 3 & 2 & 3 & 3 & 5 & 5 & 4 \\
& 7 & 6 & 7 & 5 & 4 & 7 & 6 & 7 \\
\hline
\end{tabular}

The inVANETs-based intersection control algorithm consists of two parts: the vehicleTask and the controllerTask. The following representation state $_{i}$ which designate the state of the vehicle which has the state $I D L E$, when the vehicle is outside the area of intersection, the state WAITING, when the vehicle sent a request to the controller to cross the intersection and still waiting for the controller reply to cross, the state CROSSING, which shows that the vehicle is crossing the intersection area, and finally, the state CROSSED which shows that the vehicle crossed the intersection. Lane $i$ represents the lane of the vehicle. crossingLists denotes the list of vehicles that the controller accepted their request message and is allowing them to cross the intersection. Any vehicle in that list can move and then cross the intersection area.

These five messages are exchanged between the vehicleTask and the controllerTask:

- <REQUEST, $i$, lane $i>$ : The vehicle $i$ sends this message to the controller to indicate its lane and that it wants to cross the intersection.

- <CROSS, lane, vehiclesList, duration >: message sent by the controller to order waiting vehicles in vehiclesList to cross the intersection within the specified duration.

- $\left\langle D E L A Y E D, i\right.$, lane $_{i}>$ : vehicle $i$ sends this message to inform the controller that it failed in crossing the intersection within the specified duration.

- $<N E W L A N E$, $i$, lane $_{i}>$ : vehicle $i$ moved into a new lane that conflicts with its old position.

- <CROSSED, $i>$ : vehicle $i$ informs the controller that it crossed the intersection.

\subsection{VehicleTask Operations}

In Figure 3 we show the main steps on the vehicleTask as it communicates with the controllerTask. When a vehicle $i$ enters the queuing area it sends a request message to the controller $<R E Q U E S T$, $i$, lane $e_{i}$ to the controller to get permission to cross the intersection (Step 1.1), the vehicle $i$ state updates to WAITING which means that the vehicle is waiting for the reply from the controller that gives the vehicle the access to the intersection. While the vehicle is waiting to get the access from the controller, the vehicle may transfer to another lane that is conflicting with the old position that was sent earlier to the controller, so in that case, the vehicle sends the message $<N E W L A N E$, $i$, lane $_{i}>$ to the controller to notify it about the change (step 1.5). Any vehicle $i$ can be in the intersection area while crossing or outside of it after crossing or in the queuing area, and as a result of that, the vehicle state changes correspondingly.

When any vehicle $i$ receives the message $\langle$ CROSS, vehiclesList $>$ and $i$ is in the vehiclesList, only then the vehicle can cross the intersection. It was assumed in $(\mathrm{Wu} 2014)$ that when the controller grants the cross to a list of vehicles, the controller has to wait until it receives a message from the last vehicle in the list to confirm that every vehicle crossed which can lead to delays in real life scenarios if a slow driver is driving the vehicle or a congestion happened in the intersection. To solve this problem, a duration period was added, which is set by the controller after which the vehicles that are delayed will not get access to the intersection until the controller decides based on the waiting list. When this period elapses, any vehicle $i$, which was delayed and was not able to cross the intersection, this vehicle will receive the delayed message $\langle D E L A Y E D$, vehiclesList $\rangle$ in order to be 
notified by the controller that this vehicle has been changed to the waiting state again. In addition, in order to solve the situation where a vehicle may have changed its lane to a conflicting one after sending the REQUEST message, in the CROSS message, the lane that was given access to the intersection was added.

In (Elhadef 2015), (Wu 2014), it was assumed that the last vehicle in the vehicles list to cross the intersection is the vehicle that will notify the controller by sending a message noted as RELEASE. When taking into consideration that the vehicles may get delayed and aren't able to cross the intersection in the specified period of time, the algorithm was changed to make the vehicle send the CROSSED message to the controller when it crosses the intersection (step 1.6), and in that way the controller will know which vehicles already crossed the intersection, and which vehicles were delayed because they will send the DELAYED message.

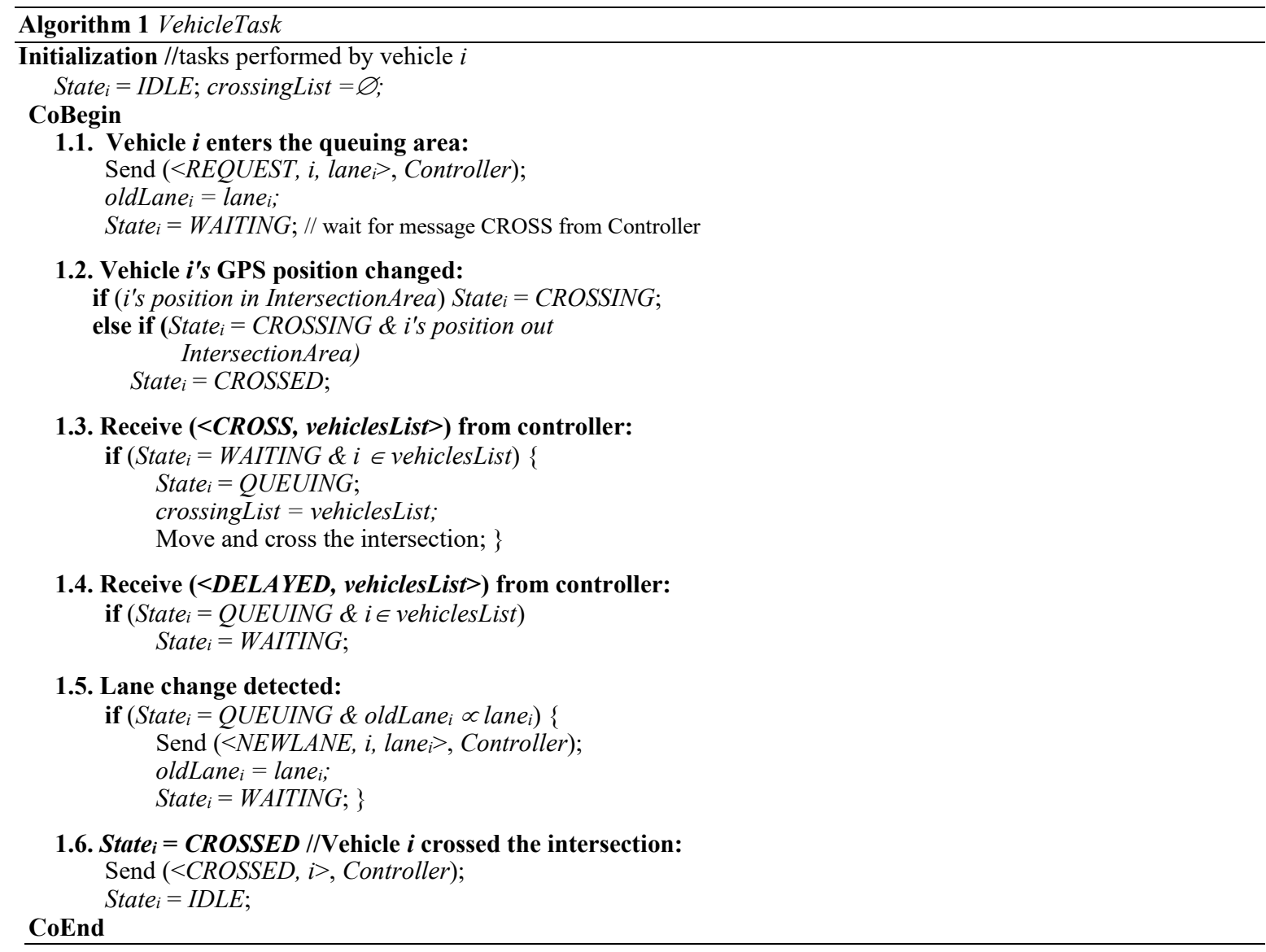

Figure 3. The vehicleTask inVANETs-based intersection control algorithm

\subsection{ControllerTask Operations}

The controllerTask main steps are shown in Figure 4 locks $_{i}$ represents the set of lanes that are going to be locked for vehicle $i$ (refer to Table 1) and locked represents the set of lanes that are already locked by the controller at any certain point of time. When the controller receives $<$ REQUEST, $i$, lane $e_{i}>$ from the vehicle $i$ which is sending a request in order to cross the intersection (step 2.1), first, the controller examines if the set of lanes to be locked for this vehicle are already locked by the controller then at that time $i$ is added to the crossingList of vehicles which are given the access to the intersection, and then the controller broadcasts the cross message <CROSS, crossingList> to the vehicles in the crossingList to notify them that they are able to cross the intersection. However, if the controller didn't lock any lane then vehicle $i$, and the other vehicles on concurrent lanes with vehicle $i$ can cross the intersection. Else, in that case, the controller adds vehicle $i$ to the waitingList. The controller also sets the crossingTimer to certain duration, and hence giving the access to the intersection for a period that is limited. Also, the controller may receive the new lane message $\langle N E W L A N E$, $i$, newLane > from the vehicle that changed its lane. Then the controller updates its information. If the vehicle sending this message changed its lane to a conflicting one with the old one, which has been granted access to the intersection and is 
in the crossingList, then the vehicle $\mathrm{i}$ is moved from the crossingList to the waitingList. (Step 2.2). When the controller receives the message crossed $\langle C R O S S E D, i>$ which informs the controller that the vehicle $i$ crossed the intersection, and after that the controller updates the information in the crossingList, and when it the crossingList becomes empty then the controller gives access to the intersection to the first vehicle in waitingList.

The controller uses the crossingTimer for the implementation of fairness property. The fairness property means that any vehicle will have the chance to cross the intersection after a finite amount of time (step 2.4). When the timer finishes, the controller examines first if there are waiting vehicles. If there is, the controller checks if the vehicles that were given access to cross the intersection crossed the intersection or not. If some of these vehicles were delayed the controller will send them the delayed message $\langle D E L A Y E D$, crossingList $>$ to notify these vehicles that they need to wait up until the next round. In order to manage the situation of a vehicle being currently in the intersection, then the timer waits for another crossingDuration before giving the access to other vehicles to cross the intersection. If there are no vehicles waiting then the controller extends the duration for crossing to delayed vehicles.

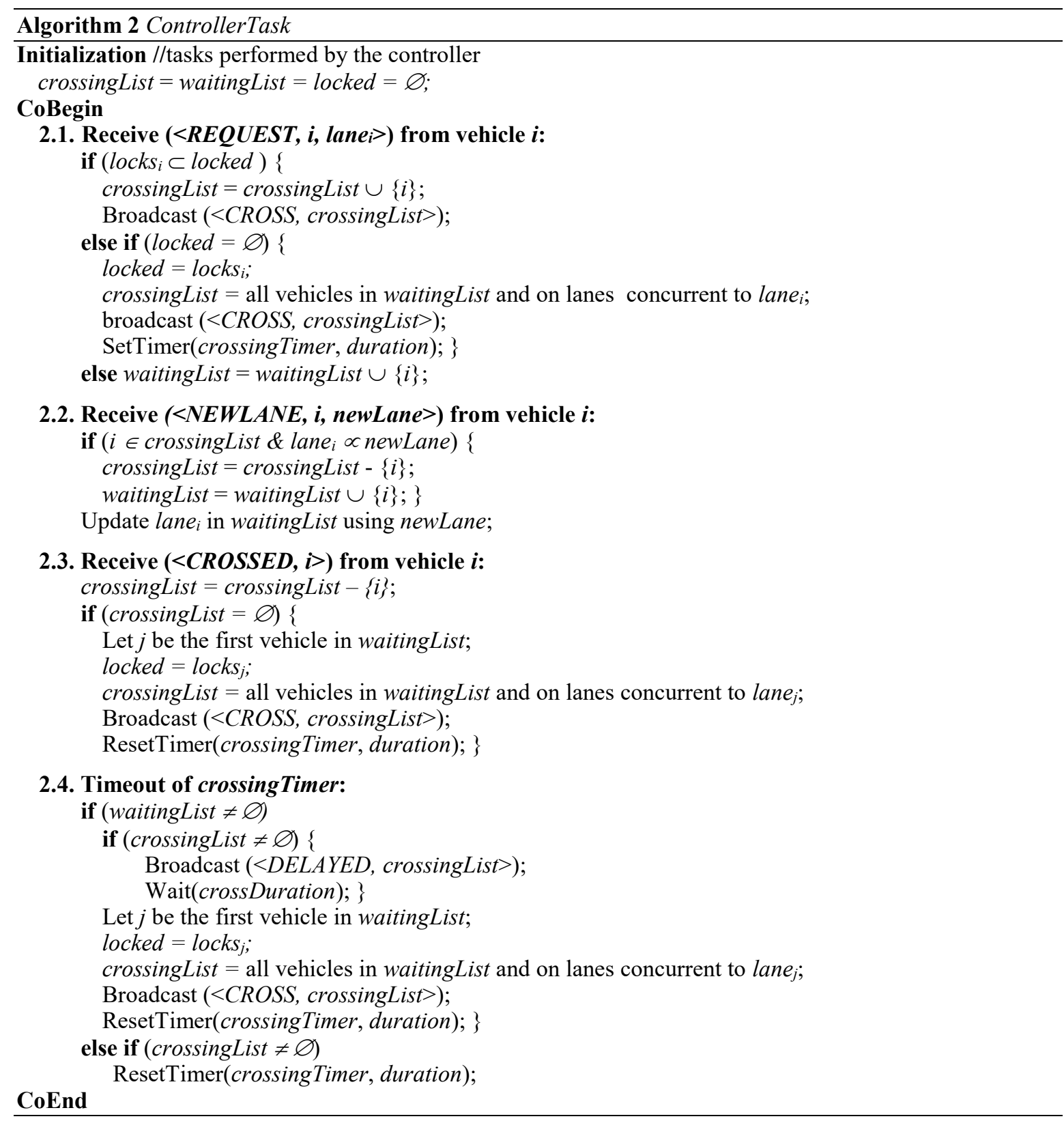

Figure 4. The controllerTask inVANETs-based intersection control algorithm 


\section{APPLICATION OF AN ADAPTABLE inVANETS-BASED INTERSECTION TRAFFIC CONTROL ALGORITHM ON A REAL-LIFE INTERSECTION IN ABU DHABI}

In this section, we will discuss the application of the algorithm on a real-life intersection in Abu Dhabi. Mainly, Abu Dhabi City has intersection has around 4 lanes in each road, so the intersection will have around 15 lanes, as seen in Figure 5, and Figure 6. Therefore, when applying the locks table, we will get this locks table for 16 lanes.

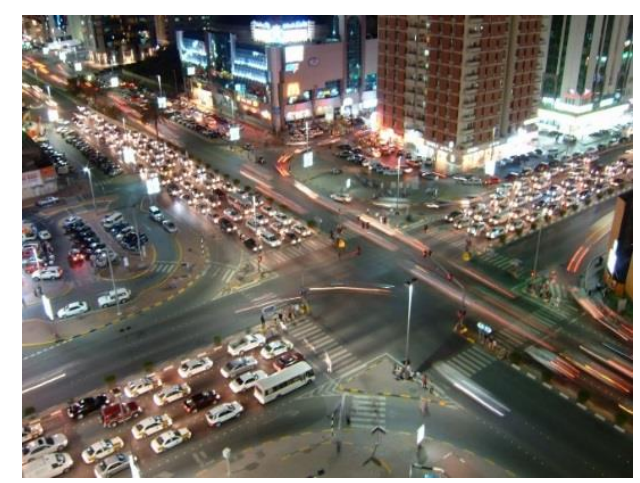

Figure 5. An intersection in Abu Dhabi

Each lane will lock its lane with 4 conflicting lanes, but we need to take in consideration that some of the lanes are "twins", which means that these lanes are identical which means they are concurrent with each other, and concurrent with the same lanes, as well as conflict with the same lanes on the road. Referring to Table 2, In order to solve this issue, the twin lanes will have the same locks, and when 1 lane of the twin requests to lock it will mean the other twin lane is concurrent and shares the same locks. For example, when a vehicle $i$ in $l_{0}$ requests the access to the intersection from the controller, and the controller locks $l_{0}$ locks, it means that it will allow a vehicle $j$ on $l_{l}$ to cross as well since it shares the same exact locks with 10 which are $l_{10}, l_{11}, l_{014}$, and $l_{15}$. Similarly, like Table 1. If a vehicle $i$ is concurrent with the lane that the controller already has its locks it will get access to the intersection, for example like $l_{0} \approx l_{2}$. Also, if vehicle $i$ is on a conflicting lane then the controller will request it to wait. For example, like $l_{l} \propto l_{4}$.

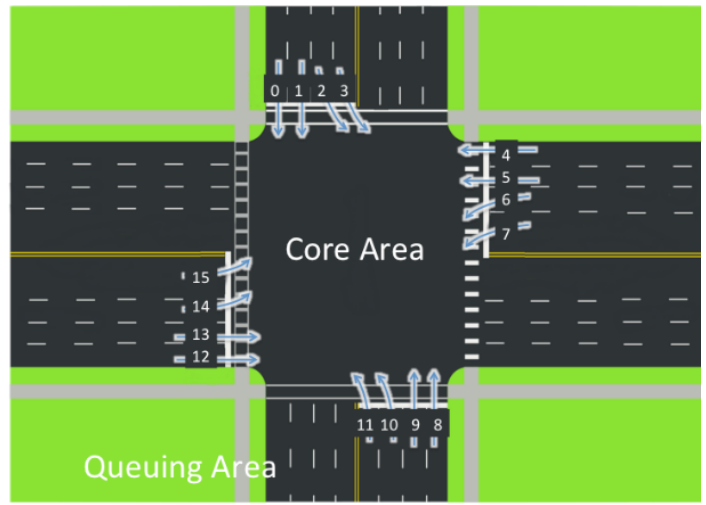

Figure 6. View of an intersection in Abu Dhabi

\section{SIMULATION RESULTS}

In order to check the performance of the developed algorithm, we analyzed simulation results generated on different metrics that depend on mainly on the traffic of vehicles in the simulation. 


\subsection{Simulation Environment}

We developed our algorithm based on VEINS, an open source framework for the simulation of vehicular networks in which it uses Omnet++, and SUMO to simulate the vehicular networks. We used a map of a real intersection in the city of Abu Dhabi, and it was created using Open Street Map, which is a geographic data tool. In Figure 7, we can see the traffic in the simulation generated.

We ran our algorithm on nine simulations generated with different scenarios. In figure 8 , we can see that the vehicles are distributed in an almost non-uniform fashion on four different roads.

Table 2. The lane locking scheme for an intersection in Abu Dhabi

\begin{tabular}{|l|c|c|c|c|c|c|}
\hline Vehicle's lane & $\mathbf{0}$ & $\mathbf{1 , 2}$ & $\mathbf{3 , 4}$ & $\mathbf{5}$ & $\mathbf{6 , 7}$ & $\mathbf{8 , 9}$ \\
\hline & 0 & $(1,2)$ & $(3,4)$ & 5 & $(6,7)$ & $(8,9)$ \\
Lanes to be locked & 6 & 13 & 8 & 11 & 3 & 1 \\
& 7 & 14 & 9 & 12 & 4 & 2 \\
& & 18 & 16 & & 18 & 13 \\
& & 19 & 17 & & 19 & 14 \\
\hline Vehicle's lane & $\mathbf{1 0}$ & $\mathbf{1 1 , 1 2}$ & $\mathbf{1 3 , 1 4}$ & $\mathbf{1 5}$ & $\mathbf{1 6 , 1 7}$ & $\mathbf{1 8 , 1 9}$ \\
\hline & 10 & $(11,12)$ & $(13,14)$ & 15 & $(16,17)$ & $(18,19)$ \\
Lanes to be locked & 16 & 3 & 6 & 1 & 8 & 3 \\
& 17 & 4 & 7 & 2 & 9 & 4 \\
& & 8 & 18 & & 13 & 11 \\
& & 9 & 19 & & 14 & 12 \\
\hline
\end{tabular}

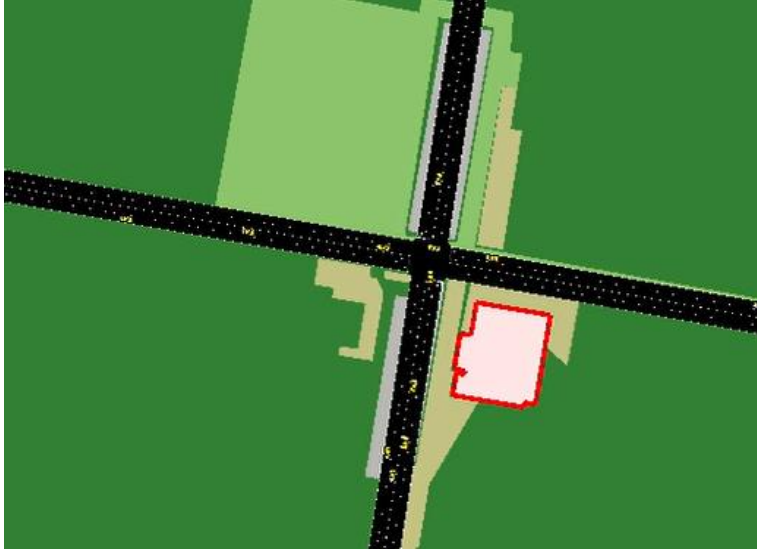

Figure 7. Simulation on Omnet++ and SUMO

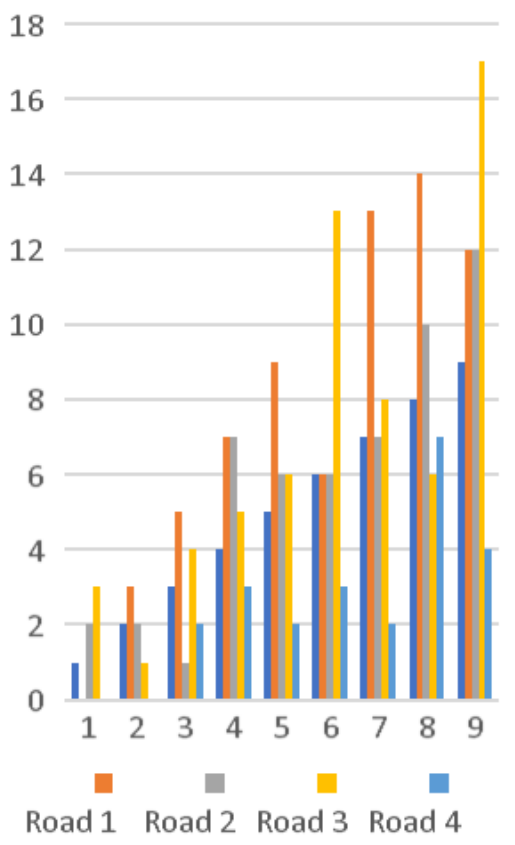

Figure 8. Distribution of vehicles on roads on scenarios

\subsection{Simulation Metrics}

The size of the intersection that we are using is $200 \mathrm{~m} * 200 \mathrm{~m}$. The transmission reaches up to $510 \mathrm{~m}$, and we use IEEE $802.11 \mathrm{p}$ for the communication in the network since it is a wireless communication protocol environment that are vehicular. The algorithm was used on different scenarios having different numbers of vehicles ranging between 0 to 50 . The simulation time varies depending on the number of vehicles as it is from the range of $51 \mathrm{~s}$ to 150 s. We will show the results based on these performance metrics: 
- Average message cost: Average number of messages that is sent by each vehicle from the time it entered the queuing area till it crosses the intersection.

- Average queue length: Average number of waiting vehicles in queue area.

- Average waiting time: Average duration that each vehicle stays from the time it entered the queuing area till it crosses the intersection.

- System throughput: Number of vehicles that crosses the intersection in each minute.

\subsection{Average Message Cost}

In Figure 9, we can see the different messages sent on the network whether they are from the vehicles or the controller. Each vehicle is allowed to send one request message to the controller, and then the controller decides which vehicles that will get the permission to cross based on the locking mechanism and send a cross message to the vehicles who are allowed to cross based on that mechanism. The vehicles then cross the intersection and send the crossed message to the controller. In addition, the controller has a crossing timer that will allow the waiting vehicles to get a chance to cross the intersection; therefore, in that case, it will send the delayed message to the vehicles in order to give the permission to other vehicles to cross the intersection.

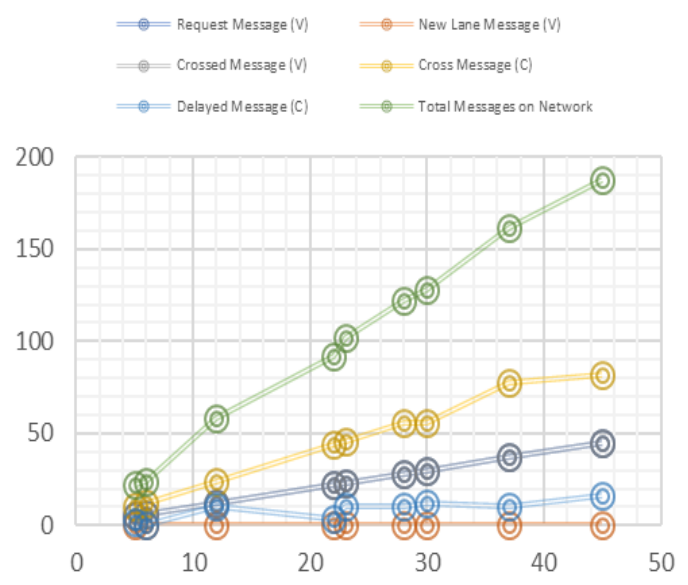

Figure 9. Messages on the Network

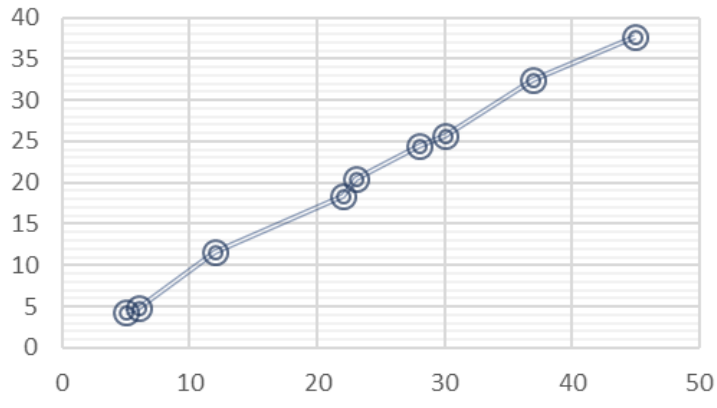

Figure 10. Average Message Cost

In figure 10, we can see the average message cost in the network, we can notice as we increase the number of vehicles in each simulation, the average messages increases steadily.

\subsection{Average Queue Length}

We can see in figure 11, the Average Queue Length, we notice that the queue length depends on the scenario itself, so we can see it might increase or decrease depending on the real traffic generated by the scenarios we have. We can notice that there is no steady increase in this figure.

\subsection{Average Waiting Time}

In figure 12, the simulation results show that the Average Waiting Time depends on the Average Queue length of the scenario, so if the scenario has a longer queue, the vehicles will have a longer waiting time, so the waiting time does not increase steadily, but instead increases or decrease based on the traffic generated by the scenario. 


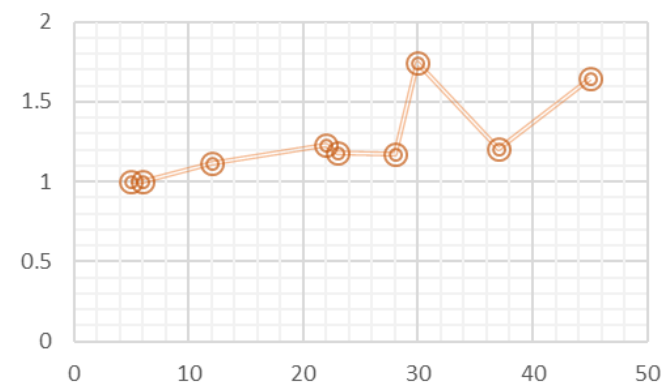

Figure 11. Average Queue Length

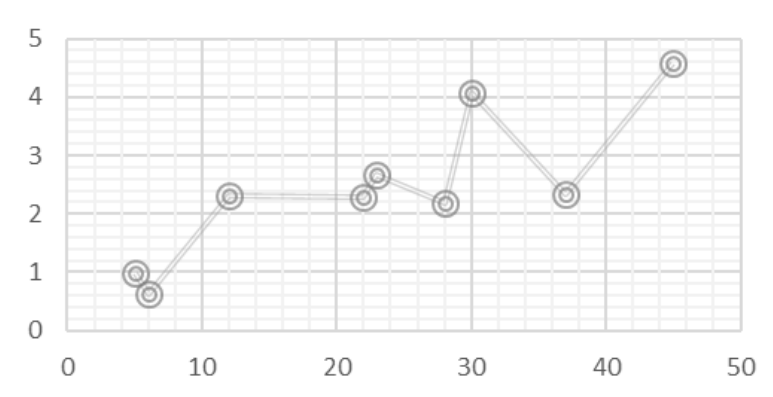

Figure 12. The Average Waiting Time

\subsection{System Throughput}

The simulation results shown in figure 13 display the system throughput throughout the different simulations we have generated. When we notice previous graphs, we can see that the throughput is in relation with the Average Queue Length and the Average Waiting Time, the scenarios that have longer queues, and waiting times have less system throughput, but in general there's an increase in the system throughput using the algorithm we created.

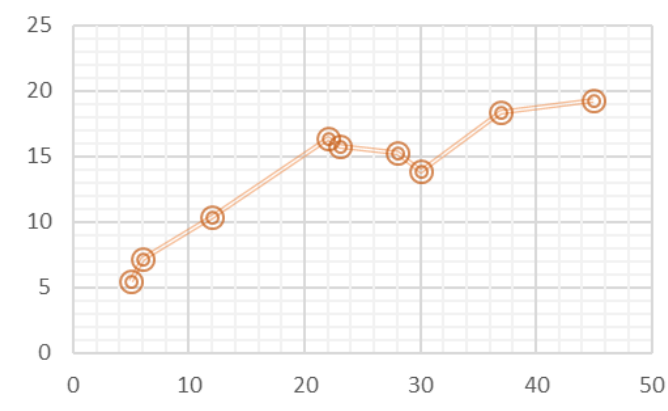

Figure 13. System Throughput 1/M

\section{CONCLUSION}

In this paper, we discussed developed novel adaptable centralized intersection control for ITS (Intelligent Transportation Systems) which depends on inVANETs (Intelligent Vehicular ad-hoc Networks). The intersection control based on inVANETs contains two main parts: the vehicleTask, and the controllerTask. These two tasks exchange message and cooperate to make the vehicles able to cross the intersection without suffering from any type of conflicts.

In addition, we also, applied the algorithm on an intersection in Abu Dhabi, and explained the improvements necessary in order to be applied with no issues, since the intersections in Abu Dhabi are much larger than the intersection proposed in the algorithm.

We also performed extensive performance evaluation of the algorithm and displayed the results for this evaluation. The traffic in the scenarios were in a non-uniform fashion, we showed statistics involving the message cost, the queue length, the waiting time, and the throughput in each scenario of these different scenarios.

\section{ACKNOWLEDGEMENT}

This work is supported by ADEK Award for Research Excellence (A2RE) 2015 and office of Research and Sponsored Programs, Abu Dhabi University. 


\section{REFERENCES}

Bhatt V. (2010). "Group Mutual Exclusion in O(log n) RMR", Proc. 29th ACM SIGACT-SIGOPS Symp. Principles of Distributed Computing (PODC-10), pp.45 -54.

Bulgannawar S. (1995). "A Distributed K-Mutual Exclusion Algorithm", Proc. 15th Int. Conf. Distributed Computing Systems (ICDCS).

Chen X. (2002). "Real-Coded Genetic Algorithm for Signal Timings Optimization of a Signal Intersection", Proc. First Int. Conf. Machine Learning and Cybernetics, pp. 1245-1248. IEEE Press, Beijing.

Dresner K. (2008). "A Multiagent Approach to Autonomous Intersection Management", J. Artificial Intelligence Research, vol. 31, no. 1, pp.591 -656.

Elhadef, M. (2015). “An Adaptable inVANETs-Based Intersection Traffic Control Algorithm,” IEEE Int. Conference on Computer and Information Technology; Ubiquitous Computing and Communications; Dependable, Autonomic and Secure Computing; Pervasive Intelligence and Computing, no. 6, pp. 2387 - 2392.

Gokulan B.P. (2010). "Distributed Geometric Fuzzy Multiagent Urban Traffic Signal Control", IEEE Trans. Intelligent Transportation Systems, vol. 11, no. 3, pp.714 -727.

Lee J. (2012). "Development and Evaluation of a Cooperative Vehicle Intersection Control Algorithm Under the Connected Vehicles Environment", IEEE Trans. Intelligent Transportation Systems, vol. 13, no. 1, pp.81 -90.

Lertworawanich P. (2011). "A New Multiobjective Signal Optimization for Oversaturated Networks", IEEE Trans. Intelligent Transportation Systems, Vol. 12(4), pp.967 -976.

Qiao J. (2011). "Two-Stage Fuzzy Logic Controller for Signalized Intersection", IEEE Trans. Systems, Man and Cybernetics-Part A: Systems and Humans, vol. 41, no. 1, pp.178 -184.

Raravi G. (2007). "Merge Algorithms for Intelligent Vehicles", Proc. GM R\&D Workshop, pp.51 -65.

Srinivasan D. (2006). "Neural Networks for Real-Time Traffic Control System", IEEE Trans. Intelligent Transportation Systems, Vol. 7(3), pp.261 -272.

Tubaishat M. (2009). "Wireless Sensor Networks in Intelligent Transportation Systems", Wireless Comm. and Mobile Computing, vol. 9, no. 3, pp.287 -302.

Wei Ni (2014). “A Message Efficient Intersection Control Algorithm Based on VANETs," Internet of Vehicles Technologies and Services, Lecture Notes in Computer Science Volume 8662, pp 31-41.

Wu W.G. (2015). "Distributed Mutual Exclusion Algorithms for Intersection Traffic Control," IEEE Transactions on Parallel \& Distributed Systems, Vol.26 (1), pp. 65-74.

Zhao D. (2012). "Computational Intelligence in Urban Traffic Signal Control: A Survey", IEEE Trans. Systems, Man, and Cybernetics-Part C: Applications and Reviews, Vol. 42(4), pp.485 -494.

Zhou B. (2010). "Adaptive Traffic Light Control in Wireless Sensor Network-Based Intelligent Transportation System", Proc. IEEE Vehicular Technology Conf. Fall.

Milans V. et al (2010). "Controller for Urban Intersections Based on Wireless Communications and Fuzzy Logic", IEEE Trans. Intelligent Transportation Systems, vol. 11, no. 1, pp.243 -248.

Glaser S. et al (2010). "Maneuver-Based Trajectory Planning for Highly Autonomous Vehicles on Real Road with Traffic and Driver Interaction", IEEE Trans. Intelligent Transportation Systems, vol. 11, no. 3, pp.589 -606.

Hasan S. and M. Elhadef (2018). A Citywide Distributed inVANETs-Based Protocol for Managing Traffic. In Proc. of the 13th International Conference on Future Information Technology (FutureTech 2018), Salerno, Italy.

Saeed I. and M. Elhadef (2017). A Distributed inVANETs-Based Intersection Traffic Control Algorithm. In Proc. of the 9th International Conference on Computer Science and its Applications (CSA 2017), Taichung, Taiwan.

Baras S., I. Saeed, H. A. Tabaza, and M. Elhadef (2017). VANETs-Based Intelligent Transportation Systems: An Overview. In Proc. of the 11th Int. Conference on Multimedia and Ubiquitous Engineering, Seoul, Korea. 